Originalien

Hautarzt 2020 · 71:613-623

https://doi.org/10.1007/s00105-020-04616-0

Online publiziert: 3. Juni 2020

(c) Der/die Autor(en) 2020

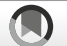

check tor
updates

\section{Hintergrund und Fragestellung}

Neben der häufigen Berufskrankheit BK-Nr. 5101 („Schwere oder wiederholt rückfällige Hauterkrankungen“) kommen in der Dermatologie selten andere arbeitsbedingte Hauterkrankungen vor, die unter andere BK-Nummern fallen (• Tab. 1).

Unter der Nr. 3101 der Anlage 1 zur BKV (Berufskrankheiten-Verordnung) sind Krankheiten erfasst, die von Mensch zu Mensch übertragbar sind [4] (weitere Kriterien: s. - Tab. 2). Die Verdachtsanzeige einer Berufskrankheit erfolgt bei der BK-Nr. 3101 mittels BKAnzeige (Formular F 6000).

Für die Anerkennung einer BKNr. 3101 ist erforderlich, dass der Versicherte einer - der versicherten Tätigkeit innewohnenden - Infektionsgefahr besonders ausgesetzt war [6]. Es handelt sich um eine offene BerufskrankheitenBezeichnung: Erfasst werden alle Arten von berufsbedingten Infektionskrankheiten, z. B. Tuberkulose, Hepatitis A-E, HIV („human immunodeficiency virus")/AIDS („acquired immune deficiency syndrome"), MRSA (Methicillin-resistenter Staphylococcus aureus), SARS-CoV2/COVID-19 und andere ([30]; • Abb. 1).

\author{
Cornelia Erfurt-Berge ${ }^{1}$ Anne Schmidt ${ }^{2}$ Irena Angelovska ${ }^{1,3} \cdot$ Vera Mahler $^{1,4}$ \\ ${ }^{1}$ Hautklinik Universitätsklinikum Erlangen, Erlangen, Deutschland \\ ${ }^{2}$ Büro für Berufsdermatologie, Begutachtung und Betriebsberatung, Nürnberg, Deutschland \\ ${ }^{3}$ Hautarztpraxis Wolfratshausen, Wolfratshausen, Deutschland \\ ${ }^{4}$ Paul-Ehrlich-Institut, Langen, Deutschland
}

\title{
Beruflich erworbene MRSA- Besiedelung in der berufsdermatologischen Begutachtung (BK-Nr. 3101)
}

\section{Auswertung der DGUV BK-Dokumentation und Gutachtenfall mit arbeitsbedingter MRSA-getriggerter atopischer Dermatitis}

Die Infektion mit einer übertragbaren Krankheit erfüllt regelmäßig auch die Voraussetzungen des Unfallbegriffs, weil das auslösende Ereignis die einmalige Ansteckung (innerhalb einer Arbeitsschicht) ist [30]. Diese ist jedoch rückblickend häufig nicht zu ermitteln, insbesondere bei zunächst klinisch stumm verlaufenden Erkrankungen [30].

Ziel der Arbeit war es, die besonderen medizinischen und versicherungsrechtlichen Aspekte einer beruflichen MRSAKolonisation darzustellen am Beispiel eines eindrucksvollen Gutachtenfalles und der Auswertung der BK-Verdachtsanzeigen (BK-Nr. 3101) der gewerblichen Berufsgenossenschaften und Unfallversicherungsträger.

\section{MRSA und BK-Nr. 3101}

MRSA ist weltweit als Problemkeim in Krankenhäusern und Pflegeeinrichtungen anerkannt [25]. Pflegeheime stellen ein Umfeld für den Erwerb und die Verbreitung von MRSA dar. Risikofaktoren für den MRSA-Erwerb sind chronische Erkrankungen, offene Wunden und häufige Anwendung antimikrobieller Substanzen, wie sie bei Bewohnern eines Pflegeheims gehäuft vorkommen können. Erwerb einer MRSA-Besiedelung ist auch im Rahmen einer beruflichen Tätigkeit in der Pflege möglich.

Die Prävalenz einer MRSA-Kolonisation für Patienten in Kliniken und Pflegeeinrichtungen in Europa variiert aufgrund unterschiedlicher Screening- und Präventionsmaßnahmen zwischen 1 und $20 \%$ [10]. Sowohl die Resistenzraten als auch die Prävalenz des Methicillin-resistenten Staphylococcus aureus (MRSA) bleiben in Deutschland seit Jahren auf einem stabilen Niveau [24]. Bei Bewohnern von Pflegeheimen in Deutschland wird die Prävalenz mit ca. $3 \%$ angegeben [17]. Die durchschnittliche MRSA-Prävalenz im Gesundheitsdienst liegt zwischen 1,1 und $5,4 \%[1,12]$.

Ein beruflich bedingter Erwerb der MRSA-Kolonisation ist bei Berufstätigen im Gesundheitswesen als wahrscheinlich anzusehen, wenn andere MRSARisikofaktoren wie Herkunft aus Endemiegebieten, längere stationäre Krankenhausaufenthalte, beruflicher Kontakt $\mathrm{zu}$ landwirtschaftlicher Tiermast mit Einsatz von Antibiotika oder außerberuflicher Kontakt zu Patienten mit chronischer Pflegebedürftigkeit ausgeschlossen werden können [23]. Für die Anerkennung einer BK-Nr. 3101 ist grundsätzlich der Nachweis einer Infektionsquelle erforderlich. In Ausnah- 
Tab. 1 Berufskrankheiten gemäßAnlage 1 zur Berufskrankheitenverordnung (Stand 01.01.2015) die mit Hauterscheinungen einhergehen können

\section{Berufskrankheiten mit möglicher Hautbeteiligung}

5101 Schwere oder wiederholt rückfällige Hauterkrankungen ${ }^{a}$

5102 Hautkrebs durch Ruß, Rohparaffin, Teer, Anthrazen, Pech oder ähnliche Stoffe

5103 Plattenepithelkarzinome oder multiple aktinische Keratosen der Haut durch natürliche UV-Strahlung

1108 Erkrankungen durch Arsen oder seine Verbindungen

1310 und Erkrankung durch Halogenkohlenwasserstoffe oder halogenierte Alkyl-, Arylverbin1311 dungen, Halogene

$1314 \quad$ Erkrankungen durch p-tertiäres Butylphenol

1315 Erkrankungen durch Isocyanate

2402 Erkrankungen durch ionisierende Strahlen

3101 Infektionskrankheiten, wenn der Versicherte im Gesundheitsdienst, in der Wohlfahrtspflege oder in einem Laboratorium tätig oder durch eine andere Tätigkeit der Infektionsgefahr in ähnlichem Maße besonders ausgesetzt war

3102 Von Tieren auf Menschen übertragbare Krankheiten ${ }^{\text {b }}$

4301 Durch allergisierende Stoffe verursachte obstruktive Atemwegserkrankungen ${ }^{\mathrm{a}}$

4302 Durch chemisch-irritative oder toxisch wirkende Stoffe verursachte Atemwegserkrankungen

${ }^{\text {a} A l l e r g i e i n d u z i e r t e ~ a n a p h y l a k t i s c h e ~ R e a k t i o n e n ~ n a c h ~ L a t e x s e n s i b i l i s i e r u n g ~ s i n d ~ i n ~ D e u t s c h l a n d ~ n a c h ~}$ überwiegend betroffenem Organsystem subsumiert unter BK-Nr. 5101 und/oder 4301

bDurch Zeckenbiss übertragene Krankheiten sind in Deutschland subsumiert unter BK-Nr. 3102 Im Gegensatz zu BK-Nr. 4301, die sich aufgrund der gemeinsamen immunologischen Pathophysiologie als Systemerkrankung (an Atemwegen und Haut) manifestieren kann (wie z. B. Typ-I-Allergie auf Naturgummilatex), ist dies bei der Einwirkung chemisch-irritativ oder toxisch wirkender Arbeitsstoffe, die ein irritatives Asthma verursachen, nicht der Fall. Dennoch verfügen chemisch-irritative oder toxisch wirkende atemwegsreizende Stoffe (Gefahrenhinweise für die Gesundheitsgefahren: H335 „Kann die Atemwege reizen") nicht selten bei entsprechender Exposition (in Abhängigkeit von Dauer und Intensität der Einwirkung auf die Haut) auch über hautreizende Wirkung (H315 „Verursacht Hautreizungen"), die als Begleiterscheinungen bei entsprechender Koexposition möglich sind

\section{Tab. 2 Kriterien einer BK(Berufskrankheit)-Nr. 3101 (nach [4])}

\section{Kriterien für BK-Nr. 3101}

1 Von Mensch zu Mensch übertragbare Krankheiten fallen grundsätzlich dann unter die BK-Nr. 3101, wenn sie bei Versicherten auftreten, die infolge der Ausübung ihrer beruflichen Tätigkeit in bestimmten Bereichen einer gegenüber der allgemeinen Bevölkerung wesentlich erhöhten Infektionsgefahr ausgesetzt sind

2 Dies trifft hauptsächlich auf das Personal in stationären oder ambulanten medizinischen Einrichtungen der Human- und Zahnmedizin, in wohlfahrtspflegerischen Einrichtungen und Laboratorien zu

3 Außerdem können in diesen Bereichen kurzfristig tätige Personen (z. B. Wartung, Instandsetzung oder Entsorgung) betroffen sein

$4 \quad$ Ein Risiko in ähnlichem Maße kann auch bei Tätigkeiten in der Gentechnik, Biotechnologie, in Abwasser- und Kläranlagen bestehen

5 Gemeinsam ist allen Fällen, dass die Erregerübertragung ein einmaliges, punktuelles Ereignis darstellt, das häufig im Nachhinein nicht mehr ermittelt werden kann

6 Als Ergebnis der Erregerübertragung resultiert hierbei lokale oder systemische Ausbreitung mit oder ohne Krankheitssymptome mefällen können für ausgewählte Tätigkeitsbereiche Beweiserleichterungen ${ }^{1}$ gelten. MRSA-kolonisierte Berufstätige in Gesundheits- und Pflegeberufen sind zumeist asymptomatisch, stellen aber als Vektor ein wichtiges Bindeglied in der Übertragung von MRSA dar. Die MRSAKolonisation (d. h. klinisch stumme Besiedelung von Haut und Schleimhäuten mit Bakterienvermehrung, aber ohne Auftreten von Infektionszeichen) ist von einer apparenten MRSA-Infektion mit Einschränkungen des Gesundheitszustandes zu unterscheiden [23].

Nach der bisherigen Beurteilungspraxis werden klinisch stumme MRSA-Kolonisationen nicht als BK-Nr. 3101 anerkannt, sondern nur im Falle klinisch manifester Infektionszeichen [15]. Im Gegensatz zu Kontaktekzemen, die $95 \%$ der BK-Nr. 5101 ausmachen, sind dabei charakteristischerweise weder der Verlauf noch die Lokalisation streng tätigkeitsabhängig. Stellt sich die Hautkrankheit als Folge einer MRSA-Infektion dar, liegen die Voraussetzungen einer BK-Nr. 5101 nicht vor. Es ist eine BK-Nr. 3101 zu prüfen [30].

Im Gegensatz zur klinisch stummen MRSA-Besiedelung/Kolonisation der Haut oder Schleimhäute des $\mathrm{Na}$ sen-Rachen-Raums als Bestandteil der Hautflora, bei der kein krankhafter/ regelwidriger Zustand erkennbar ist, kommt es bei einer MRSA-verursachten Infektionskrankheit, die als BK-Nr. 3101 anzuerkennen ist, $\mathrm{zu}$ einem physisch regelwidrigen Zustand im Sinne einer Gesundheitsstörung (z.B. MRSA-assoziierte Infektionen oder Superinfektion an läsionaler Haut, im Bereich der Harnwege, des Mittelohres oder der Gelenke, ausgehend von einer lokalen Infektion

\footnotetext{
1 Der Gesetzgeber hat in $\S 9$ Abs. 3 BKV für die Beurteilung des Kausalzusammenhangs eine Beweiserleichterung festgelegt: Erkranken Versicherte, die infolge der besonderen Bedingungen ihrer versicherten Tätigkeit in erhöhtem Maße der Gefahr der Erkrankung an einer in derRechtsverordnung nach Absatz 1 genannten Berufskrankheit ausgesetzt waren, an einer solchen Krankheit und können Anhaltspunkte für eine Verursachung außerhalb der versicherten Tätigkeit nicht festgestellt werden, wird vermutet, dass diese infolge der versicherten Tätigkeit verursacht worden ist.
} 
Hautarzt 2020 - 71:613-623 https://doi.org/10.1007/s00105-020-04616-0

(c) Der/die Autor(en) 2020

\section{Erfurt-Berge $\cdot$ A. Schmidt $\cdot$ I. Angelovska $\cdot$ V. Mahler}

Beruflich erworbene MRSA-Besiedelung in der berufsdermatologischen Begutachtung (BK-Nr. 3101). Auswertung der DGUV BK-Dokumentation und Gutachtenfall mit arbeitsbedingter MRSA-getriggerter atopischer Dermatitis

\section{Zusammenfassung}

Hintergrund. Von Mensch zu Mensch übertragene Infektionskrankheiten können eine Berufskrankheit(BK)-Nr. 3101 bedingen, wenn sie bei Versicherten auftreten, die infolge der Ausübung ihrer Arbeitstätigkeit in bestimmten Bereichen einer gegenüber der allgemeinen Bevölkerung wesentlich erhöhten Infektionsgefahr ausgesetzt sind. Ziel der Arbeit. Es erfolgt die Darstellung der besonderen medizinischen und versicherungsrechtlichen Aspekte einer beruflichen MRSA-Kolonisation am Beispiel eines Gutachtenfalles und Auswertung der BK-Verdachtsanzeigen (BK-Nr. 3101) der gewerblichen Berufsgenossenschaften und Unfallversicherungsträger.

Patienten und Methoden. Die Geschäftsergebnisse und BK-Dokumentation der gewerblichen Berufsgenossenschaften und
Unfallversicherungsträger 2007 bis 2012 wurden zur BK-Nr. 3101 ausgewertet sowie das Patientenkollektiv berufsdermatologischer Begutachtungsfälle 2007 bis 2012 der Hautklinik Universitätsklinikum Erlangen zum Vorliegen von Mensch zu Mensch übertragener Infektionskrankheiten der Haut retrospektiv untersucht.

Ergebnisse. Von Mensch zu Mensch übertragene Infektionskrankheiten der Haut sind in der berufsdermatologischen Begutachtung selten. Von den 2007 bis 2012 erfassten BKVerdachtsanzeigen waren 2,6\% BK-Nr. 3101Verdachtsfälle; $4,2 \%$ aller anerkannten BKen entfielen auf BK-Nr. 3101-Fälle, darunter 9 Fälle von MRSA (Methicillin-resistenter Staphylococcus aureus). Eine symptomlose MRSA-Besiedlung wird im Gegensatz zur manifesten Infektionskrankheit nicht als BK-
Nr. 3101 anerkannt. Bakterielle Superantigene können eine atopische Dermatitis (AD) triggern. Im Einzelfall kann eine AD infolge einer beruflich erworbenen MRSA-Ansteckung auftreten und eine BK-Nr. 3101 begründen. Diskussion. Ein frühzeitiger Nachweis einer MRSA-Kolonisation und Eradikation sind notwendig für eine Rehabilitation. Der Umgang mit Hauterkrankungen als Folge einer Infektionskrankheit im Berufskrankheitenverfahren wird dargelegt.

\section{Schlüsselwörter}

Stumme MRSA-Kolonisierung · Regelwidriger Gesundheitszustand · Berufskrankheitenverfahren · Berufskrankheit · Von Mensch zu Mensch übertragbare Erkrankung

\section{Occupationally acquired MRSA colonization and occupational dermatological assessments (BK-No. 3101 in the German list of Occupational Diseases). Analysis of the DGUV documentation and expert opinion on a case with work-related MRSA-triggered atopic dermatitis}

\section{Abstract}

Background. Person-to-person transmitted infectious diseases can cause occupational diseases (OD). These are subsumed as BK-No. 3101 in the German list of OD which applies for individuals with a considerably higher risk for infection as a consequence of their professional activity compared to the general population.

Objectives. The special medical and

insurance law aspects of a work-related MRSA colonization are presented using the example of an expert opinion case and an evaluation of the BK reports of suspected occupational disease (BK No. 3101) of the German Social Accident Insurance (DGUV).

Patients and methods. The BK documentation of the DGUV from 2007-2012 and the patient cohort from the Department of Dermatology, University Hospital Erlangen, presenting for expert assessment from 2007-2012 were retrospectively analysed for human-to-human transmitted infectious diseases of the skin (BKNo. 3101).

Results. Person-to-person transmission of infectious diseases of the skin is rare in the field of occupational dermatology. In the DGUV cohort, suspected BK-No. 3101 cases amounted to $2.6 \%$ of all notified cases; recognized BK-No. 3101 cases accounted for $4.2 \%$ of all recognized cases, amongst which 9 were caused by MRSA. In contrast to a symptomatic infection, an asymptomatic MRSA colonization is not being recognized as BK-No. 3101. Bacterial superantigens can trigger atopic dermatitis (AD). In particular cases, occupationally acquired MRSA can elicit $A D$ and may justify classification as an OD (BKNo. 3101).

Conclusions. Early detection of MRSA colonization and eradication are necessary for rehabilitation. Management of skin diseases due to infectious diseases within the framework of OD is presented.

\section{Keywords}

Asymptomatic MRSA colonization · Abnormal health condition - Occupational disease evaluation proceedings . Occupation diseases. Communicable disease zu einer systemischen Ausbreitung mit Auftreten tief liegender Abszesse oder Befall von Lunge oder Herz) [23].

\section{Patientenkollektive und Methoden}

Die bei der Deutschen Gesetzlichen Unfallversicherung (DGUV) geführte Dokumentation der Berufskrankheiten (BK-DOK) sowie die Geschäfts- und Rechnungsergebnisse der gewerblichen
Berufsgenossenschaften und Unfallversicherungsträger der öffentlichen Hand (Zeitraum 2007-2012) wurden zur BKNr. 3101 ausgewertet (dankenswerterweise zur Verfügung gestellt von Stephanie Schneider [DGUV Referat Statistik Leistungen, Berufskrankheiten, Sonderaufgaben, DGUV; Berlin]). 


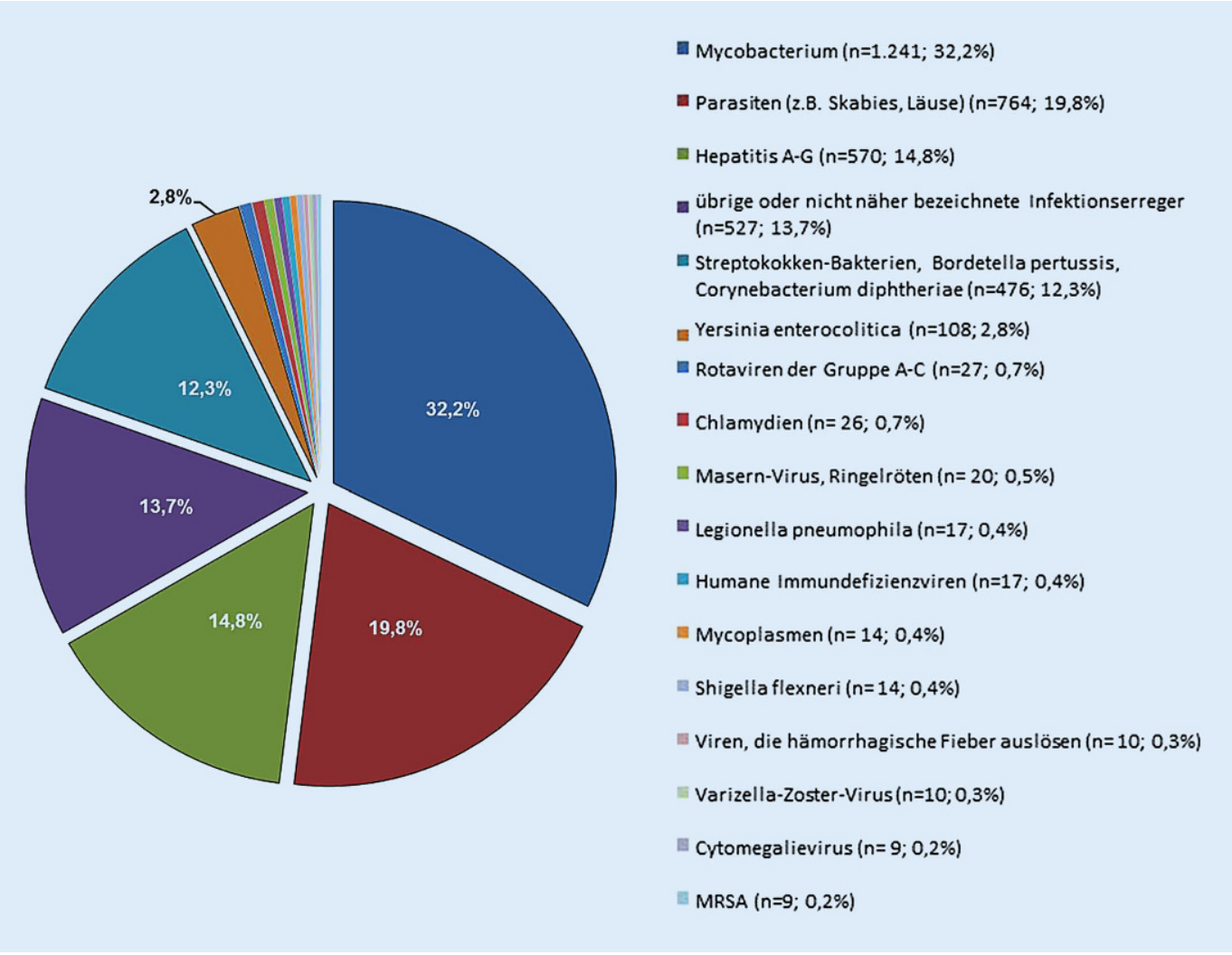

Abb. $1 \varangle$ Von Mensch zu Mensch übertragene ErregeralsBK(Berufskrankheit)auslösender Gegenstand (bei $n=3859 \mathrm{im}$ Zeitraum 2007 bis 2012 anerkannten BKen 3101 gemäß BK-DOK der DGUV [Deutsche Gesetzliche Unfallversicherung e. V.]). MRSA wird in der BK-DOK erst seit 2009 als eigener BK-auslösender Gegenstand erfasst. In der Gruppe "übrige oder nicht näher bezeichnete Infektionserreger" sind Erreger zusammengefasst, die in der BK-DOK nicht als eigener BK-auslösender Gegenstand kategorisiert sind

Zusätzlich wurde das 2007 bis 2012 nach der ärztlichen Praxis berufsdermatologisch begutachtete Patientenkollektiv der Hautklinik Universitätsklinikum Erlangen bezüglich von Mensch zu Mensch übertragener Infektionskrankheiten der Haut retrospektiv untersucht. Die Datenextraktion erfolgte dabei in pseudonymisierter Form unter Einhaltung ethischer Richtlinien im Einklang mit nationalem Recht sowie gemäß der Deklaration von Helsinki von 1975 (in der aktuellen, überarbeiteten Fassung).

\section{Ergebnisse}

\section{Daten der gewerblichen}

Berufsgenossenschaften und Unfallversicherungsträger der öffentlichen Hand

Im Zeitraum 2007 bis 2012 betrug der Anteil der angezeigten BK-Nr. 3101-Verdachtsfälle $(n=10.443) 2,6 \%$ aller angezeigten BK-Verdachtsfälle $(n=400.949)$. Die anerkannten BK-Nr. 3101-Fälle ( $n=3711$, davon 487 mit einer BK-Rente) im selben Zeitraum machten $4,2 \%$ aller anerkannten Berufskrankheiten $(n=88.447)$ aus (•Tab. 3). Die Häufigkeitsverteilung der unterschiedlichen Erreger als BK-auslösendem Gegenstand der von 2007 bis 2012 anerkannten BKNr. 3101-Fälle $(n=3859)$ ist in • Abb. 1 zusammengefasst: Zwei Drittel aller Fälle sind bedingt durch beruflich erworbene Tuberkulose, Parasiten (v. a. z.B. Skabies und Läuse) und Hepatitis A-G (- Abb. 1).

Nach aktuellen Zahlen der Geschäftsund Rechnungsergebnisse [7] wurden im Jahr 2013 von den insgesamt 71.579 Anzeigen auf Verdacht einer Berufskrankheit $n=1704 \quad(2,4 \%)$ Infektionskrankheiten unter dem Verdacht einer Berufskrankheit BK-Nr. 3101 angezeigt. Bei $n=721$ (2\%) der insgesamt 36.202 Fälle mit bestätigtem BK-Verdacht lag eine BK-Nr. 3101 vor. In Vergleich dazu betrugen die 2013 unter der BK-Nr. 5101 angezeigten Fälle $(n=24.033)$ - überwiegend mit Kontaktekzemen - 33,6\% aller angezeigten Fälle, von denen bei $n=20.643$ der berufliche Zusammenhang bestätigt wurde (entspricht $57 \%$ aller Fälle mit bestätigtem BK-Verdacht; [8]).

\section{Berufsdermatologisches}

Begutachtungskollektiv der

Hautklinik Universitätsklinikum

Erlangen

Das oben genannte Patientenkollektiv berufsdermatologischer Begutachtungsfälle umfasste 2007 bis $2012 n=129$ Gutachten, von denen nur 1 Fall $(0,77 \%)$ die BK-Nr. 3101 betraf. Die Fragestellungen des Gutachtenauftrages sind in • Tab. 4 zusammengefasst.

In diesem speziellen Fall (•Tab. 5; - Abb. 2) wurde die Anerkennung einer MRSA-assoziierten Ekzemerkrankung infolge einer beruflich erworbenen MRSA-Ansteckung als BK-Nr. 3101 gutachterlicherseits empfohlen und durch den Unfallversicherungsträger anerkannt, da es sich im Gegensatz zu einer klinisch stummen MRSA-Kolonisation um eine klinisch manifeste MRSA-assoziierte Erkrankung der Haut als regelwidrigem Gesundheitszustand handelte.

Berufsdermatologische Beurteilung: Im speziellem Gutachtenfall einer Altenpflegerin (•Tab. 5) fällt gemäß Gutachtenauftrag die Fragestellung nach Folgen der MRSA-Erkrankung im dermato- 
Tab. 3 BK(Berufskrankheit)-Nr. 3101 im Berufskrankheitengeschehen (2007 bis 2012; Geschäftsund Rechnungsergebnisse der gewerblichen Berufsgenossenschaften und Unfallversicherungsträger der öffentlichen Hand)

\begin{tabular}{|c|c|c|c|c|c|c|}
\hline & \multicolumn{3}{|l|}{ BK 3101} & \multicolumn{3}{|l|}{ Insgesamt } \\
\hline & $\begin{array}{l}\text { Verdachts- } \\
\text { anzeigen }\end{array}$ & $\begin{array}{l}\text { Anerkann- } \\
\text { te BKen }\end{array}$ & $\begin{array}{l}\text { Darunter: } \\
\text { Neue BK- } \\
\text { Renten }\end{array}$ & $\begin{array}{l}\text { Verdachts- } \\
\text { anzeigen }\end{array}$ & $\begin{array}{l}\text { Anerkann- } \\
\text { te BKen }\end{array}$ & $\begin{array}{l}\text { Darunter } \\
\text { Neue BK- } \\
\text { Renten }\end{array}$ \\
\hline 2007 & 2515 & 735 & 107 & 61.150 & 13.383 & 4123 \\
\hline 2008 & 1507 & 462 & 97 & 60.736 & 12.972 & 4312 \\
\hline 2009 & 1689 & 499 & 76 & 66.951 & 16.078 & 6643 \\
\hline 2010 & 1493 & 579 & 64 & 70.277 & 15.461 & 6123 \\
\hline 2011 & 1645 & 641 & 72 & 71.269 & 15.262 & 5407 \\
\hline 2012 & 1594 & 795 & 71 & 70.566 & 15.291 & 4924 \\
\hline Gesamt & 10.443 & 3711 & 487 & 400.949 & 88.447 & 31.532 \\
\hline
\end{tabular}

logischen Fachgebiet nicht unter BKNr. 5101, sondern unter BK-Nr. 3101 (Infektionskrankheiten, wenn der Versicherte im Gesundheitsdienst, in der Wohlfahrtspflege oder in einem Laboratorium tätig oder durch eine andere Tätigkeit der Infektionsgefahr in ähnlichem Maße besonders ausgesetzt war). Eine im Vorfeld der dermatologischen Begutachtung durchgeführte gewerbeärztliche Beurteilung empfahl die Anerkennung als BK-Nr. 3101.

Zum Zeitpunkt der Begutachtung wurde eine generalisierte, akute atopische Dermatitis mit erneut nachgewiesener MRSA-Besiedelung der Haut diagnostiziert. Es bestand mit an Sicherheit grenzender Wahrscheinlichkeit eine beruflich erworbene MRSA-Ansteckung bei deutlich erhöhtem Risiko im Rahmen der beruflichen Tätigkeit als Altenpflegehelferin. Für die atopische Dermatitis ist eine Exazerbation der Hautveränderungen durch MRSA-Kolonisation der Haut in der Literatur beschrieben [28].

Die über den Zeitpunkt des Eintretens der Arbeitsunfähigkeit als Altenpflegehelferin fortdauernde MRSA-Besiedelung der Haut stellt im vorliegenden Fall eine wesentliche Teilursache der Erstmanifestation der atopischen Dermatitis im 60. Lebensjahr und der zunächst bestehenden Therapieresistenz der atopischen Dermatitis dar, die nach erfolgreicher MRSA-Sanierung vollständig und nachhaltig abheilte.

Patienten mit atopischer Hautdiathese zeigen eine höhere Suszeptibilität für die Besiedelung mit Staphylococcus (S.) aureus aufgrund einer reduzierten Ex- pression antimikrobieller Peptide [31]. Toll-like-Rezeptoren (TLR), besonders TLR-2, erkennen Zellwandbestandteile von S. aureus [27]. Monozyten von Patienten mit einem heterozygoten TLR-2Polymorphismus, der mit einer schwereren klinischen Krankheitsmanifestation assoziiert ist, produzieren signifikant mehr IL(Interleukin)-6 und IL-12 nach TLR-2-Aktivierung [27]. Verschiebungen in der bakteriellen Prädominanz einzelner Spezies (S. aureus, S. epidermidis, Streptococcus, Propionibakterien und Korynebakterien) führen bei Patienten mit atopischer Dermatitis zu einem vermehrten Auftreten von Krankheitsschüben [22]. Hierbei kommt es pathophysiologisch durch eine erhöhte Proteaseaktivität und einen erhöhten $\mathrm{pH}$ Wert zu einer eingeschränkten Barrierefunktion der Haut, verursacht durch eine Reduktion der natürlichen Feuchthaltefaktoren („moisturizing factors“) [19]. Die pH-Wert-Erhöhung ermöglicht eine gesteigerte Adhäsion und Proliferation von Staphylokokken auf der Hautoberfläche [17]. Es konnte gezeigt werden, dass Hautkontakt zu von Staphylococcus aureus produzierten extrazellulären $\mathrm{Ve}$ sikeln eine Rolle in der Pathogenese der atopischen Dermatitis spielt [18].

Bis zum Zeitpunkt der Begutachtung wurden MRSA-Sanierungsmaßnahmen nicht konsequent bis zur erfolgreichen Eradikation durchgeführt. Bei therapieresistenter atopischer Dermatitis wurde aus gutachterlicher Sicht eine MRSAEradikation als relevantem Ekzemtrigger empfohlen, zusätzlich eine stadiengerechte dermatologische Therapie der exogen getriggerten atopischen Dermatitis [32].

Nach Anerkennung als Berufskrankheit BK-Nr. 3101 wurde seitens des gesetzlichen Unfallversicherungsträgers ein stationäres Heilverfahren veranlasst mit dem Therapieziel der erfolgreichen MRSA-Eradikation. Während des stationären Aufenthaltes wurden intensiviert lokalantiseptische Maßnahmen (- Tab. 6) über 1 Woche durchgeführt, und eine vollständige MRSA-Eradikation wurde erzielt. Die vorher über Jahre therapieresistenten ekzematösen Hautveränderungen heilten nach der MRSAEradikation vollständig und dauerhaft ab. Drei Jahre nach erfolgreicher Sanierung ist der Hautzustand der Patientin unter blanden rückfettenden Maßnahmen weiterhin erscheinungsfrei. Die berufliche Tätigkeit als Altenpflegerin wurde von der hauterscheinungs- und MRSAfreien Versicherten nicht mehr aufgenommen aufgrund einer Freistellung seitens des Arbeitgebers über 6 Monate bis zum Erreichen des Rentenalters.

Die Minderung der Erwerbsfähigkeit (MdE) durch die MRSA-verursachte Gesundheitsstörung wurde im vorliegenden Gutachtenfall zum Zeitpunkt der Begutachtung bei noch bestehender MRSABesiedelung und dadurch getriggerter atopischer Dermatitis ab dem Zeitpunkt des erstmaligen MRSA-Nachweises auf $25 \%$ geschätzt. Die Einschätzung der MdE bei BK-Nr. 3101 orientiert sich dabei an den objektivierbaren Funktions- und Leistungseinschränkungen: Aufgrund der MRSA-getriggerten atopischen Dermatitis waren alle Feuchtberufe auf dem allgemeinen Arbeitsmarkt als verschlossen anzusehen sowie alle Gesundheits- und Pflegeberufe mit engem Kontakt zu Pflegebedürftigen mit chronischen Erkrankungen.

Nach Wiedereintritt der Arbeitsfähigkeit ohne verbleibende Funktionseinschränkungen beträgt die $\mathrm{MdE} 0 \%$ [30].

\section{Diskussion}

Staphylococcus aureus besiedelt die Haut bei $60-100 \%$ der AD-Patienten im Vergleich zu 5-30\% der gesunden Kontrollen [21]. Des Weiteren weisen 10-30\% 
Tab. 4 Fragestellungen eines Zusammenhangsgutachtens zur Beurteilung des Vorliegens einer BK (Berufskrankheit) 3101

\section{Gutachtenfragestellungen}

1. Wie ist der Hautbefund am Untersuchungstag?

2. Ist die MRSA-Erkrankung Ursache oder wesentlich mitwirkende Teilursache der bei der Versicherten bestehenden unter 1. genannten Hauterkrankung? Ist eine Abgrenzung zwischen dem Verschlimmerungsanteil und der bereits vorbestehenden Hauterkrankung möglich, ggf. inwieweit? [Falls nein, war sie es früher, ggf. inwieweit?]

3. Wie sind die Folgen der MRSA-Erkrankung auf dem dermatologischen Fachgebiet?

4. Welche hiervon unabhängigen Erkrankungsfolgen bestehen?

5. Wie ist die Minderung der Erwerbsfähigkeit (MdE) zum Untersuchungstag auf hautfachärztlichem Gebiet aufgrund der MRSA-Einwirkung einzuschätzen?

6. Wie ist die MdE ab dem Tag nach Ende der ersten Arbeitsunfähigkeit, nach dem Versicherungsfalltag bis zum Tag vor der Untersuchung einzuschätzen?

7. Ist in absehbarer Zeit eine Besserung zu erwarten und bis wann voraussichtlich?

8. Welche Heilmaßnahmen halten Sie für erforderlich, um die volle Wiederherstellung bzw. weitere Besserung der Erwerbsfähigkeit zu erreichen?

MRSA Methicillin-resistenter Staphylococcus aureus

der von AD-Patienten isolierten S.aureus-Stämme Methicillin-Resistenz (MRSA) auf. Atopische Dermatitis ist eine multifaktorielle Erkrankung, bei der nach derzeitigem pathogenetischem Verständnis [21] der Kolonisation mit S. aureus eine ungünstige Einwirkung zwischen S.-aureus-Infektion und ADExazerbation zukommt durch Induktion von TSLP (,thymic stromal lymphopoietin“) und Th2/Th17-Entzündung. Alle S.aureus-Stämme exprimieren Superantigene wie Staphylokokken-Enterotoxin (SE) A, SEB, SEC, SED und toxisches Schocksyndrom-Toxin-1 (TSST-1) [21]. Hochgradig abnormale und komplexe Muster von Superantigenen werden bei mehr als $80 \%$ der von AD-Patienten isolierten S. aureus gefunden. Staphylokokken-Superantigene aktivieren polyklonale T-Zellen und anschließend eine T-Zell-vermittelte Entzündung in AD-Läsionen durch Bindung an „major histocompatibility complex class II molecules" auf dendritischen Zellen und T-Zell-Rezeptoren $\beta$-Ketten auf T-Zellen ohne antigene Peptidpräsentation. Insbesondere SEB erhöht die IL-31Expression und führt zur Hemmung der Differenzierung von Keratinozyten und zur Unterdrückung der FilaggrinExpression [21].

Im Berufskrankheitengeschehen allgemein und speziell in der berufsdermatologischen Begutachtung sind von Mensch zu Mensch übertragbare Infek- tionskrankheiten (BK-Nr. 3101) im Vergleich zu Erkrankungen der BK-Nr. 5101 selten: In der berufsdermatologischen Begutachtung überwiegen Kontaktekzeme, die unter die BK-Nr. 5101 fallen. Von Mensch zu Mensch übertragene Infektionskrankheiten können eine BK 3101 bedingen, wenn sie bei Versicherten aus dem Bereich des Gesundheitsdienstes, der Wohlfahrtspflege oder Laboratorien auftreten. Bei diesen Infektionserkrankungen kann es sich um eine Hautkrankheit handeln, bei der die BK-Nr. 3101 auch dann anzuerkennen ist, wenn die besonderen Voraussetzungen einer BKNr. 5101 nicht gegeben sind. Die Voraussetzungen für eine BK-Nr. 3101 und der Umgang damit im Berufskrankheitengeschehen unterscheiden sich wesentlich von denen der BK-Nr. 5101 und sind selbst unter erfahrenen Dermatologen zum Teil wenig bekannt, weshalb eine Mindermeldung möglich erscheint.

Während in der Gesamtstatistik der DGUV (Deutsche Gesetzliche Unfallversicherung e. V.) im Zeitraum 2007 bis 2012 BK-Nr. 3101-Verdachtsfälle mit $n=10.4432,6 \%$ aller angezeigten Verdachtsfälle und mit $n=3711$ Fällen 4,2\% aller anerkannten Berufskrankheiten (davon 9 [0,2\%] mit MRSA) ausmachten, lag im untersuchten berufsdermatologischen Begutachtungskollektiv der Hautklinik Universitätsklinikum Erlangen die Fragestellung im selben Zeitraum nur 1-mal vor.
In diesem beispielhaft dargestellten Gutachtenfall (• Tab. 5) wurde die Anerkennung einer MRSA-assoziierten Ekzemerkrankung infolge einer beruflich erworbenen MRSA-Ansteckung als BKNr. 3101 empfohlen und anerkannt, da es sich im Gegensatz zu einer klinisch stummen MRSA-Kolonisation um eine klinisch manifeste MRSA-assoziierte Erkrankung der Haut als regelwidrigem Gesundheitszustand handelte, die darüber hinaus die besonderen Voraussetzungen der BK-Nr. 3101 erfüllte. Bei der Versicherten bestanden berufliche Risikofaktoren für den Erwerb einer Besiedelung mit "healthcare associated(ha)MRSA“ [25]. Eine genetische Analyse des vorliegenden MRSA-Isolates wurde nicht durchgeführt.

Nachdem vor Aufnahme der Tätigkeit als Altenpflegehelferin die Versicherte niemals an einer atopischen Dermatitis erkrankt war, ist im speziellen Fall eine Verschlimmerung als „Gelegenheitsursache" im Sinne des Sozialrechts nicht zutreffend, da diese eine vorbestehende klinisch manifeste anlagebedingte Erkrankung voraussetzen würde $[2,13]$. Der Krankheitsverlauf der AD im speziellen Fall, die vollständig und nachhaltig nach der MRSA-Sanierung sistierte, weist auf einen beruflichen Zusammenhang im Sinne der wesentlichen Teilursache hin.

MRSA gehört nicht zu den häufigen von Mensch zu Mensch übertragbaren Infektionskrankheiten, die unter BKNr. 3101 geregelt sind. Diese sind, basierend auf der Analyse des DGUVRegisters (• Abb. 1), vorwiegend Tuberkulose, parasitäre Erkrankungen und Hepatitiden. MRSA gehört zu den weniger häufigen bakteriellen Erregern, die unter bestimmten Voraussetzungen im Sinne der BK-Nr. 3101 anerkannt werden [30]. Die Prävalenzen von Staphylococcus aureus und MRSA in der Normalbevölkerung sowie der Umstand, dass MRSA gegenüber Staphylococcus aureus keine erhöhte Virulenz hat, stellen wesentliche Hindernisse bei der Anerkennung als Berufskrankheit dar. Dass die atopische Dermatitis im oben genannten Gutachtenfall durch den MRSA erst hervorgerufen wurde, ist von entschei- 
Tab. 5 Gutachtenfall einer 64-jährigen Altenpflegerin, bei der die Erstmanifestation einer atopischen Dermatitis durch beruflich erworbenen MRSA (Methicillin-resistenter Staphylococcus aureus) ausgelöst wurde

\section{Gutachtenfall}

Beruf, $\quad$ Eine 64-jährige Altenpflegerin wurde 2012 mit therapieresistenter atopischer Dermatitis (AD) und MRSA-Nachweis (Haut und Fragestellung Nasenostium) auf Veranlassung der Berufsgenossenschaft für Gesundheitsdienst und Wohlfahrtspflege zur dermatologischen (s. - Tab. 4) Begutachtung zum Vorliegen einer BK-Nr. 3101 vorgestellt

Berufsanamnese Die ausgebildete Apothekenhelferin arbeitete nach Umschulung zur Fachgehilfin für ambulante und stationäre Pflege seit über 10 Jahren als Altenpflegehelferin in einem Seniorenheim

Sämtliche Pflegeverrichtungen (Waschen, Mobilisieren, Füttern, Anziehen der Bewohner) gehörten zu den Aufgaben der Versicherten

Beim Lagern und Umbetten bestand z. T. enger Körperkontakt zu den Pflegebedürftigen

Die Tätigkeit wurde in Vollzeit (8,5 h/Tag) ausgeübt

Am Arbeitsplatz erfolgte die Anwendung der dort vorhandenen Hautschutz- und -pflegemittel etwa 2- bis 3-mal/Tag Händewaschen erfolgte 8- bis 10-mal/Tag mit der zur Verfügung gestellten Handseife, ca. 15-mal/Tag eine Händedesinfektion $6 \mathrm{~h} / \mathrm{Tag}$ wurden latexfreie medizinische Einmalhandschuhe getragen

Hinweise auf Einwirkungen von möglichen Kontaktallergenen am Arbeitsplatz fanden sich nicht, insbesondere keine Betonung der Hautveränderungen im Bereich der Hände

Die Patientin versorgte bei ihrer Tätigkeit als Altenpflegehelferin Pflegebedürftige mit nachgewiesener MRSA-Kolonisation und Pflegebedürftige mit ungeklärtem MRSA-Status

Bei bekannter MRSA-Besiedelung von Bewohnern wurden diese vom Pflegepersonal unter Anwendung von Schutzmaßnahmen versorgt: Tragen von Kopfschutzkappen, Mundschutz, Handschuhen, Schutzkitteln und Überschuhen bei Betreten des Zimmers und Pflege des betroffenen Bewohners sowie Desinfektionsmaßnahmen gemäß der in der Pflegeeinrichtung geltenden HygieneLeitlinie. Über eine neu festgestellte MRSA-Besiedelung wurde das Pflegepersonal unmittelbar durch die Vorgesetzten informiert

Es erfolgten regelmäßige hausinterne Fortbildungen über MRSA für die Mitarbeiter. Screeninguntersuchungen auf MRSA-Besiedelung wurden bei den Mitarbeitern nicht durchgeführt

Außerberufliche Risikofaktoren für den Erwerb einer MRSA-Kolonisation bestanden bei der Versicherten nicht

Bis zum Auftreten erster Hautveränderungen im Alter von 60 Jahren - 4 Jahre vor der Begutachtung - während der beruflichen Tätigkeit als Altenpflegerin war die Versicherte stets hautgesund

Ein Jahr nach Auftreten erster Hautveränderungen wurde bei der Versicherten während der Tätigkeit als Altenpflegerin erstmals eine bakteriologische Untersuchung eines Hautabstriches durchgeführt und ein MRSA (Haut und Nasenvorhof) nachgewiesen

Im Rahmen einer stationären Behandlung der atopischen Dermatitis eingeleitete MRSA-Eradikationsmaßnahmen wurden ambulant nicht fortgeführt

Im Verlauf der Erkrankung erfolgten nach erstmaligem Nachweis von MRSA im Jahr 2009 mehrfach Kontrollabstriche der Haut, die jeweils einen positiven MRSA-Nachweis zeigten

Seit dem MRSA-Nachweis 3 Jahre vor Begutachtung und 1 Jahr nach Auftreten erster Hautveränderungen wurde bei therapieresistenten ekzematösen Hautveränderungen durch den Hausarzt Arbeitsunfähigkeit festgestellt und bestand zum Zeitpunkt der Begutachtung ununterbrochen seit 3 Jahren

Atopieanamnese Insbesondere während der Kindheit bestand kein atopisches Ekzem

Während mehrfacher stationärer Aufenthalte in verschiedenen dermatologischen Kliniken wurde zu keinem Zeitpunkt eine vollständige Abheilung der atopischen Dermatitis erreicht

Es kamen topische Kortikosteroide zur Anwendung. Eine UVB-Therapie und Systemtherapie mit Ciclosporin wurden wegen Unverträglichkeit abgebrochen

Nach Begutachtung und Durchführung einer erfolgreichen MRSA-Sanierung heilte die generalisierte atopische Dermatitis vollständig und nachhaltig ab

Hautbefund Zum Zeitpunkt der Begutachtung bestehen bei der Patientin generalisierte Hauterscheinungen: feinlamellär schuppende Papeln und Plaques an beiden Wangen, der Stirn, perioral, periorbital mit ausgeprägtem Lidödem sowie ausgeprägte Lichenifikation nuchal, an den Hand- und Fingerrücken (• Abb. 2). An den Unterarmbeugeseiten erythematöse Plaques mit feinlamellärer Schuppung und Erosionen, an den Flanken sowie beiden Beinen teils flächig konfluierende krustöse Erosionen

16 Punkte im Erlanger Atopie-Score (Hinweis auf eine ausgeprägte atopische Hautdiathese) [9]

Keine Anzeichen für eine lokale oder systemische MRSA-Infektion (im Sinne von Abszessen, Weichteilinfektionen, Phlegmonen oder Organinfektionen) 


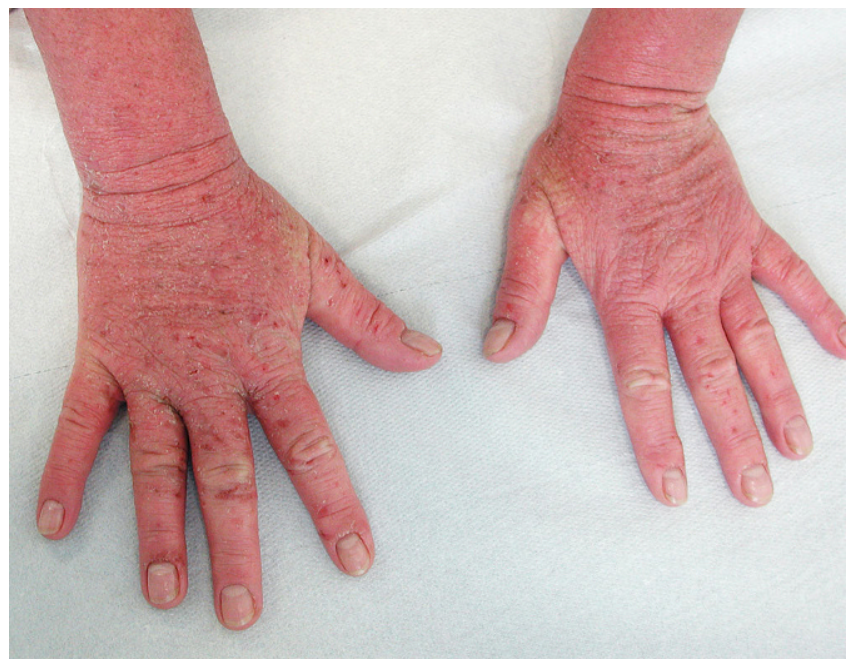

Abb. $2 \triangleleft$ Hautveränderungen der Hände der Versicherten. MRSA (Methicillin-resistenter Staphylococcus aureus) auf Haut und Schleimhaut im Nasenostium war seit 3 Jahren wiederholt nachweisbar dender Bedeutung für die Anerkennung als Berufskrankheit in diesem Fall.

Eine individuelle Betrachtung des Einzelfalles ist immer erforderlich. Zusammenfassend können in ähnlichen Fällen zur Anerkennung als BK-Nr. 3101 folgende 2 Möglichkeiten diskutiert werden:

- Anerkennung als infektverursachte Dermatitis vom atopischen Typ, die durch die beruflich verursachte Besiedelung mit MRSA verursacht wurde. Diese Möglichkeit würde der engen ursächlichen Verknüpfung der MRSA-Besiedelung mit der Dermatitis Rechnung tragen;

- Anerkennung des regelwidrigen Zustandes einer beruflich verursachten Besiedelung der Haut mit MRSA als BK 3101 dem Grunde nach und als mittelbare Folge eine atopische Dermatitis. Dieses Vorgehen wäre rechtskonform zu einem Unfall, dem die BK 3101 in der rechtlichen Bewertung naheliegt und bei dem unmittelbare und mittelbare Folgen anzuerkennen und ggf. zu behandeln und zu entschädigen sind. Für Versicherte hätte diese zweite Vorgehensweise zur Folge, dass alle etwa zusätzlich auftretenden Probleme durch die MRSA-Besiedelung anerkannt würden, nicht aber unabhängig auftretende atopische Hauterscheinungen.

Eine „normale“ atopische Dermatitis ohne beruflich erworbene MRSA-Besie- delung -, die einem krankheitstypischen Spontanverlauf folgt, ist abzugrenzen. Für Patienten mit atopischer Dermatitis wurden erhöhte Kolonisationsraten mit S. aureus von etwa $60 \%$ beschrieben [3]. Vornehmlich handelt es sich jedoch um sog. „community-acquired“ MRSA [29]. Eine vorbestehende berufsunabhängige AD kann durch eine solche Kolonisation als „Gelegenheitsursache“ verschlimmert werden. Kürzlich wurde eine signifikant stärkere Ekzemausprägung im Eczema Area Severity Index (EASI) bei ADPatienten mit multiresistenter S.-aureusBesiedelung im Vergleich zu AD-Patienten mit Methicillin-sensibler S.-aureusBesiedelung beschrieben $(p<0,01)$ [20].

Gesetzliche Richtlinien bezüglich eines Beschäftigungsverbotes bei MRSATrägerstatus bestehen nicht. Bei MRSATrägerstatus ist von Tätigkeiten in Gesundheits- und Pflegeberufen mit Kontakt zu chronisch Kranken oder Pflegebedürftigen mit offenen Wunden abzuraten. Auf Empfehlung des Robert KochInstitutes sollte zur Prävention einer Weiterverbreitung von MRSA Personal mit Keimträgerstatus bis zur nachgewiesenen Sanierung keine Patienten behandeln und pflegen [23]. In Zusammenarbeit mit dem Arbeitgeber, Betriebsarzt und der zuständigen Gesundheitsbehörde muss im individuellen Fall über eine mögliche innerbetriebliche Umsetzung entschieden werden. Da sich MRSA von der sensiblen Staphylokokken-Variante nicht durch seine Virulenzeigenschaften unterscheidet, ist bei gesunden
Menschen ohne Hautläsionen oder chronische Krankheiten keine erhöhte Infektionsgefahr mit MRSA festzustellen, und es bestehen daher per se keine beruflichen Einschränkungen von MRSA-Trägern.

Da bei der pflegerischen Tätigkeit in einem Seniorenwohnheim enger Kontakt zu Bewohnern mit chronischen Erkrankungen besteht, kann unter Anwendung von $₫ 31$ des Infektionsschutzgesetzes ein „berufliches Tätigkeitsverbot “ [5] für solche Tätigkeiten von der zuständigen Behörde - im Falle von Berufstätigen im Gesundheitswesen das zuständige Gesundheitsamt - ausgesprochen werden, was jedoch mangels Meldung/Meldepflicht in der Praxis kaum erfolgt. Im Hinblick auf den allgemeinen Arbeitsmarkt ist daher bei dermatologischen Erkrankungen mit MRSA-Besiedelung der Einsatz im Gesundheits- und Pflegebereich mit direkter Patientenbetreuung für MRSA-Träger (bis zur erfolgreichen Dekolonisierung) als verschlossen anzusehen. Bei einer ausschließlichen Besiedelung der Nase (das ist bei MRSA-Trägern bei Weitem der häufigste Fall) sollte hingegen eine Risikoeinschätzung erfolgen: Üblicherweise wird das Personal bis zum Ende der Sanierung nicht in Hochrisikobereichen (z. B. der Neugeborenenstation) eingesetzt. Bei guter Compliance mit der Händehygiene (Desinfektion vor und nach Tätigkeit am Patienten, Tragen von Handschuhen, Tragen eines Mund-Nasen-Schutzes) können ggf. auch während der Sanierungsphase z. B. Tätigkeiten am Patienten in der Altenpflege oder auch der ambulanten Pflege durchgeführt werden.

Ein zeitiger MRSA-Nachweis [16] und frühzeitige und konsequente MRSA-Sanierung [23] sind anzustreben. Ulzera (Dekubitus, arterieller, kombinierter Dekubitus/arterielle Ulzera, nicht anderweitig spezifiziert), Typ-II-Diabetes mellitus (DM) und atopische Dermatitis (AD) sind signifikant mit dem Vorliegen von MRSA verbunden [7]. In einem ambulant MRSA-sanierten dermatologischen Patientenkollektiv konnten 37,5\% der Patienten mit 5-tägigen antiseptischen Maßnahmen erfolgreich saniert werden [26]. Im Durchschnitt nahm die erfolgreiche Sanierung 12,97 $( \pm 7,6)$ Tage in Anspruch. Bei 37,5\% der Patienten war eine systemische Antibiose notwendig [26]. Die bis 
Tab. 6 Klinikinterne Standards zur Dekolonisierung, die im speziellen Fall zur Eradikation führten, im Einklang mit Empfehlungen des Robert Koch-Institutes [23]

\section{Eradikationsmaßnahmen}

Mupirocin-Nasensalbe 2- bis 3-mal täglich über 3 bis 5 Tage

Antiseptische Mundspüllösung mit nachgewiesener Wirksamkeit gegen MRSA 3-mal täglich

Haut- und Haardesinfektion mit antiseptisch wirksamen Waschlösungen mit nachgewiesener Wirksamkeit gegen MRSA 1-mal täglich

Wechsel von Wäsche, Kleidung und Bedarfsutensilien täglich

Vorliegende dekolonisierungshemmende Faktoren beseitigen (z. B. chronische Wunden adäquat behandeln, Fremdkörper, wie z. B. Katheter, entfernen)

Durchführen von Kontrollabstrichen an 3 unterschiedlichen Tagen nach 1-wöchiger Eradikation +3 Tagen ohne Maßnahmen

Empfohlene Kontrollabstriche 3, 6, und 12 Monate nach erfolgreicher Dekolonisierung

MRSA Methicillin-resistenter Staphylococcus aureus

9/2018 gültige DEGAM (Deutsche Gesellschaft für Allgemeinmedizin und Familienmedizin e. V.) S1-Handlungsempfehlung „MRSA - eine Handreichung für Hausärzte Teil 2: Therapie/Sanierung“" empfiehlt eine 5-tägige Sanierung und führt aus: „Offene Wunden, Hauterkrankungen oder liegende Zugänge wie z.B. Blasenkatheter oder PEG gefährden den Erfolg der Sanierung und sollten zuvor behandelt bzw. beseitigt werden. Wenn die Beseitigung nicht möglich ist, muss die Indikation zur Sanierung in Frage gestellt werden: nur bei erhöhter Gefahr einer Ausbreitung (z.B. im Altenpflegeheim, bei Dialysepflichtigkeit oder vor geplanten weiteren stationären Aufenthalten) ist ein Sanierungsversuch sinnvoll, da hierdurch eine Keimlastsenkung erzielt werden kann" [14].

Zusammenfassend zeigt sich, dass bei dermatologischen Erkrankungen eine 5-tägige ambulante Sanierungsdauer teilweise nicht ausreicht, ggf. für eine erfolgreiche Sanierung wie im vorliegenden Fall eine stationäre Behandlungsmaßnahme erforderlich werden kann.

Wird nach erfolgreicher Sanierung (Sanierungsmaßnahmen s. - Tab. 6) in aufeinanderfolgenden Kontrollabstrichen an 3 verschiedenen Tagen kein MRSA mehr nachgewiesen, ist eine Aufnahme der Tätigkeit in der direkten Patientenbetreuung wieder möglich. Weitere Kontrollen sind 3, 6 und 12 Monate nach Therapieende $\mathrm{zu}$ veranlassen [23].

Die Kosten dieser Maßnahmen werden bei anerkannter BK-Nr. 3101 als Leistungen nach Eintritt des Versiche- rungsfalls (Heilbehandlung), deren Anspruch sich direkt aus dem SGB (Sozialgesetzbuch) VII ableitet, durch den gesetzlichen Unfallversicherungsträger übernommen. Nachgewiesene MRSAassoziierte Krankheitssymptome und Gesundheitsstörungen im Sinne eines regelwidrigen Gesundheitszustandes wie im vorliegenden Fall der vorübergehend MRSA-getriggerten atopischen Dermatitis - stellen die Grundlage zur Entscheidung über Leistungen nach dem SGB VII dar [30].

Anders als im oben genannten Gutachtenfall wird ein MRSA sich in aller Regel bei Beschäftigten im Gesundheitswesen auf vorbestehende berufsbedingte Handekzeme, für die der Sachverhalt einer BK 5101 vorliegt, aufpfropfen. In diesem Fall sollte entsprechend ein Hautarztbericht erstattet werden. Die mit dem Hautarztverfahren für Dermatologen geschaffenen Möglichkeiten sollten genutzt werden, gerade auch bei superinfiziertem Handekzem im Gesundheitswesen.

Umfragen unter Betriebsärzten in Deutschland ergaben, dass derzeit noch sehr große Unterschiede zwischen verschiedenen Pflegeeinrichtungen bezüglich des Umgangs mit MRSA-kolonisierten Mitarbeitern herrschen [11].

\section{Fazit für die Praxis}

- Bei von Mensch zu Mensch übertragenen Infektionskrankheiten (z. B. durch Viren oder Bakterien) ist es wichtig, an einen möglichen Zusammenhang mit der Arbeitstätigkeit des Betroffenen zu denken, da diese eine BK-Nr. 3101 bedingen können, wenn der Versicherte im Gesundheitsdienst, in der Wohlfahrtspflege oder in einem Laboratorium tätig oder durch eine andere Tätigkeit der Infektionsgefahr in ähnlichem Maße besonders ausgesetzt war.

- In diesem Fall ist eine BK-Meldung an den Unfallversicherungsträger erforderlich.

- Bei besonders suszeptiblen Personen mit atopischer Hautdiathese als Anlage kann eine berufliche erworbene MRSA-Besiedelung der Haut als eine rechtlich wesentliche Teilursache zur Erstmanifestation einer hierdurch zunächst therapieresistenten atopischen Dermatitis führen, weshalb frühzeitiger MRSA-Nachweis und -Eradikation sinnvoll und erforderlich für eine medizinische Rehabilitation sind.

\section{Korrespondenzadresse}

Prof. Dr. med. Vera Mahler

Paul-Ehrlich-Institut

Paul-Ehrlich-Str. 51-59, 63225 Langen,

Deutschland

vera.mahler@pei.de

Danksagung. Die Autoren danken Frau Stephanie Schneider (Deutsche Gesetzliche Unfallversicherung [DGUV] - Spitzenverband der gewerblichen Berufsgenossenschaften und der Unfallversicherungsträger der öffentlichen Hand; Referat Statistik - Leistungen, Berufskrankheiten, Sonderaufgaben, DGUV; Berlin) für die Auswertungen der Dokumentation der Berufskrankheiten (BK-DOK) und der Geschäftsund Rechnungsergebnisse der gewerblichen Berufsgenossenschaften und Unfallversicherungsträger der öffentlichen Hand zur BK-Nr. 3101; Frau Claudia Drechsel-Schlund (Bezirksverwaltung Würzburg der Berufsgenossenschaft für Gesundheitsdienst und Wohlfahrtspflege, Würzburg) und Herrn Steffen Krohn (Deutsche Gesetzliche Unfallversicherung [DGUV] - Spitzenverband der gewerblichen Berufsgenossenschaften und der Unfallversicherungsträger der öffentlichen Hand; Abteilung Versicherung und Leistungen, DGUV, Berlin) für die konstruktive und fruchtbare Diskussion des Manuskripts.

Funding. Open Access funding provided by Projekt DEAL.

\section{Einhaltung ethischer Richtlinien}

Interessenkonflikt. C. Erfurt-Berge, A. Schmidt, I. Angelovska und V. Mahler geben an, dass kein Interessenkonflikt besteht. 
Für diesen Beitrag wurden von den Autoren keine Studien an Menschen oder Tieren durchgeführt. Für die aufgeführten Studien gelten die jeweils dort angegebenen ethischen Richtlinien. Für Bildmaterial oder anderweitige Angaben innerhalb des Manuskripts, über die Patienten zu identifizieren sind, liegt von ihnen und/oder ihren gesetzlichen Vertretern eine schriftliche Einwilligung vor.

Open Access. Dieser Artikel wird unter der Creative Commons Namensnennung 4.0 International Lizenz veröffentlicht, welche die Nutzung, Vervielfältigung Verbreitung und Wiedergabe in jeglichem Medium und Format erlaubt, sofern Sie den/die ursprünglichen Autor(en) und die Quelle ordnungsgemäß nennen, einen Link zur Creative Commons Lizenz beifügen und angeben, ob Änderungen vorgenommen wurden.

Die in diesem Artikel enthaltenen Bilder und sonstiges Drittmaterial unterliegen ebenfalls der genannten Creative Commons Lizenz, sofern sich aus der Abbildungslegende nichts anderes ergibt. Sofern das betreffende Material nicht unter der genannten Creative Commons Lizenz steht und die betreffende Handlung nicht nach gesetzlichen Vorschriften erlaubt ist, ist für die oben aufgeführten Weiterverwendungen des Materials die Einwilligung des jeweiligen Rechteinhabers einzuholen.

Weitere Details zur Lizenz entnehmen Sie bitte der Lizenzinformation auf https://creativecommons.org/ licenses/by-nd/4.0/deed.de.

\section{Literatur}

1. Albrich WC, Harbarth S(2008) Health-care workers: source, vector, or victim of MRSA? Lancet Infect Dis 8:289-301

2. Becker P (2016) Verschlimmerung in der gesetzlichen Unfallversicherung. Med Sachverstand 112:6-10

3. Bonness S, Szekat C, Novak N, Bierbaum G (2008) Pulsed-field gel electrophoresis of Staphylococcus arueus isolates from atopic patients revealing presence of similar strains in isolates from children and their parents. JClin Microbiol 46:456-461

4. Bundesanstalt für Arbeitsschutz und Arbeitsmedizin (2001) Merkblatt zur BK Nr. 3101: Infektionskrankheiten, wenn der Versicherte im Gesundheitsdienst, in der Wohlfahrtspflege oder in einem Laboratorium tätig oder durch eine andere Tätigkeit der Infektionsgefahr in ähnlichem Maße besonders ausgesetzt war. Merkblatt für die ärztliche Untersuchung. Bek. des BMA v. 01.12.2000, BArbBI. 1/2001, S 35

5. Bundesgesetzblatt (2000) Infektionsschutzgesetz vom 20. Juli 2000 (BGBI. I S. 1045), zuletzt durch Artikel 2 Absatz $36 \mathrm{u}$. Artikel 4 Absatz 21 des Gesetzes vom 7. August 2013 (BGBI. I S. 3154) geändert (5. Abschnitt: Bekämpfung übertragbarer Erkrankungen, §31)

6. Bundessozialgericht (2009) B 2 U 30/07 R - Urteil vom 02.04.2009 (http://dejure.org)

7. Daeschlein G, von Podewils S, Bloom T et al (2015) Risikofaktoren für eine MRSA-Kolonisation bei dermatologischen Patienten in Deutschland. JDtsch Dermatol Ges 13:1015-1022

8. DGUV (2015) Zahlen und Fakten: Berufskrankheiten-Geschehen.https://www.dguv.de/de/zahlenfakten/bk-geschehen/index.jsp. Zugegriffen: 1. Apr. 2015
9. Diepgen TL, Fartasch M, Hornstein OP (1989) Evaluation and relevance of atopic basic and minor features in patients with atopic dermatitis and in the general population. Acta Derm Venereol 144:50-54

10. Dulon M, Haamann F, Peters C et al (2011) MRSA prevalence in european healthcare settings: a review. $B M C$ Infect Dis 11:138

11. Dulon M, Haamann F, Nienhaus A (2013) Involvement of occupational physicians in the management ofMRSA-colonised healthcare workers in Germany-a survey. J Occup Med Toxicol 8:16

12. Dulon M, Peters C, Schablon A, Nienhaus A (2014) MRSA carriage among healthcare workers in nonoutbreak settings in Europe and the United States: a systematic review. BMC Infect Dis 14:363

13. Erlenkämper A (1998) Sozialrecht für Mediziner. Thieme, Stuttgart, S206-231

14. Fassbender B, Rösing C, Wecknecker K (2013) DEGAM S1-Handlungsempfehlung "MRSA - eine Handreichung für Hausärzte Teil 2: Therapie/ Sanierung"

15. Haamann F, Dulon M, Nienhaus A (2011) Berufliche MRSA-Infektionen bei Beschäftigten im Gesundheitsdienst. Arbeitsmed Sozialmed Umweltmed 46:585-589

16. Hell M, Bauer JW, Laimer M (2016) Molekulare Diagnostic von Methicillin-resistentem Staphylococcus aureus. Methoden und Effektivität. Hautarzt 67:6-15

17. Heuck D, Witte W (2003) Methicillin-resistente Staphylococcus aureus (MRSA) in deutschen Altenund Pflegeheimen - zur Situation. Epidemiol Bull 19:145-148

18. Hong SW, Kim MR, Lee EY et al (2001) Extracellular vesicles derived from Staphylococcus areus induce atopic dermatitis-like skin inflammation. Allergy 66:351-359

19. Irvine AD, McLean WHI, Leung DYM (2011) Filaggrin mutations associated with skin and allergic diseases. N Engl J Med 365:1315-1327

20. Jagadeesan S, Kurien G, Divakaran MV et al (2014) Methicillin-resistant Staphylococcus aureus colonization and disease severity in atopic dermatitis: a cross-sectional study from South India. Indian J Dermatol Venereol Leprol 80:229-234

21. Kim J,Kim BE,Ahn K, Leung DYM (2019) Interactions between atopic dermatitis and Staphylococcus aureus infection: clinical implications. Allergy Asthma Immunol Res 11:593-603

22. Kong HH, Oh J, Deming C et al (2012) Temporal shifts in the skin microbiome associated with disease flares and treatment in children with atopic dermatitis. Genome Res 22:850-859

23. KRINKO (2014) Empfehlung zur Prävention und Kontrolle von Methicillinresistenten Staphylococcus aureus-Stämmen (MRSA) in medizinischen und pflegerischen Einrichtungen. Empfehlungen der Kommission für Krankenhaushygiene und Infektionsprävention (KRINKO) am RKI. Bundesgesundheitsblatt Gesundheitsforschung Gesundheitsschutz57:696-732

24. Maechler F, Geffers C, Schwab F et al (2017) Entwicklung der Resistenzsituation in Deutschland. Med Klin Intensivmed Notfmed 112:186-191

25. Mempel M, Kerzl R, Ring J (2008) Methicillin-resistenter Staphylococcus aureus (MRSA). Relevanz in der Dermatologie. Hautarzt 59:659-665

26. Meyer V, Kerk N, Mellmann A et al (2012) Ambulante MRSA-Sanierung in der Dermatologie - Behandlungsbedarf und Versorgungsrealität. JDtsch Dermatol Ges 10:186-196
27. Niebuhr M, Langnickel J, Draing C et al (2008) Dysregulation of toll-like receptor-2 (TLR-2)induced effects in monocytes from patients with atopic dermatitis: impact of the TLR-2 R753Q polymorphism. Allergy 63:728-734

28. Ong $P$ (2014) Recurrent MRSA infections in atopic dermatitis. J Allergy Clin Immunol Pract 2:396-399

29. Ring J, Darsow U, Behrend H (2012) New trends in allergy and atopic eczema. Chem Immunol Allergy 96:113-119

30. Schönberger A, Mehrtens G, Valentin H (2010) Arbeitsunfall und Berufskrankheit. Rechtliche und medizinische Grundlagen für Gutachter, Sozialverwaltung, Berater und Gerichte, 8. Aufl. Erich Schmidt Verlag, Berlin, S701-760

31. Schröder JM (2011) Antimicrobial peptides in healthy skin and atopic dermatitis. Allergol Int 60:17-24

32. Werfel T, Aberer W, Augustin M et al (2009) Atopic dermatitis: S2 guidelines. J Dtsch Dermatol Ges 7(Suppl 1):S1-S46 


\section{Ursachen für ungünstigen Verlauf von Rachenkrebs aufgeklärt \\ Leipziger Forscher finden unabhängige prognostische Faktoren für die Wiederkehr der Krebserkrankung}

In Zeiten der Coronavirus-Pandemie fokussiert sich der Blick auf die Infektion mit SARS-CoV-2 und die Lungenerkrankung COVID-19. Andere viral bedingte Erkrankungen, die eine seit mehreren Jahren weltweite Pandemie ausgelöst haben, sind aktuell weniger im Fokus. Dazu zählt der von humanen Papillomviren (HPV) verursachte Rachenkrebs. In einer Studie der Leipziger Universitätsmedizin haben Forscher untersucht, welche Faktoren einen ungünstigen Verlauf bei Patienten mit sogenannten HPV-assoziierten Oropharynxkarzinomen prognostizieren. Die Ergebnisse sind aktuell im Fachjournal Cancer erschienen.

\section{HPV-Impfung könnte auch Oropha- rynxkarzinome verhindern}

Es handelt sich bei durch HPV verursachtem Rachenkrebs, sogenannte Oropharynxkarzinome, um eine von den Tonsillen (Mandeln) ausgehende Tumorerkrankung, die oftmals Jahrzehnte nach der Infektion mit HPV auftritt. Die ursächlichen Viren sind die gleichen HPV-Typen, die Gebärmutterhalskrebs und Analkarzinome verursachen. Die von der Ständigen Impfkommission des Robert-KochInstituts gleichermaßen für Mädchen und Jungen empfohlene HPV-Impfung sollte daher auch die Häufigkeit HPV-assoziierter Oropharynxkarzinome bei Männern und Frauen vermindern helfen. Allerdings ist gegenläufig zur abnehmenden Häufigkeit anderer Kopf-Hals-Karzinome weltweit ein Anstieg der Häufigkeit von Oropharynxkarzinomen zu verzeichnen. Als Ursache dieser Pandemie wird die sexuelle Übertragung von HPV angesehen. Die Häufigkeit HPV-assoziierter Karzinome steigt jährlich um 2,1 Prozent, während nicht-HPV-assoziierte, also durch Rauchen, Alkohol und berufliche Belastung verursachte Plattenepithelkarzinome der Kopf-Hals-Region eine um 0,4 Prozent pro Jahr fallende Inzidenz zeigen.

\section{Gute Prognosen gelten nicht für alle Patienten}

Zu HPV-assoziierten Oropharynxkarzinomen forschen Ärzte und Naturwissenschaftler der Universitätsmedizin Leipzig schon seit mehreren Jahren. Innerhalb der Krebserkrankungen der Kopf-Hals-Region gelten die HPVassoziierten Oropharynxkarzinome als eine Untergruppe, die gut auf moderne Therapien ansprechen. Sie haben auch eine bessere Prognose hinsichtlich des Überlebens der Tumorerkrankung. Handelt es sich um einen durch HPV verursachten Tumor, wird das Protein p16 vermehrt produziert. Das Vorhandensein von HPV wird in Gewebeschnitten des Tumors nur anhand dieses Markers bestimmt. „Unklar war bisher, warum eine allgemein bessere Progno- se nicht für alle Patienten mit p16-positivem Oropharynxkarzinom gilt. Denn viele Patienten erleiden trotz vollständiger Entfernung des Tumors und betroffener Lymphknoten und nachfolgender Strahlentherapie einen Rückfall“, sagt Prof. Dr. Andreas Dietz, Professor für HNO-Heilkunde an der Universität Leipzig.

Studie: Prognostische Faktoren können schlechteren Verlauf bedingen

An der HNO-Universitätsklinik Leipzig wird unter Leitung von Professor Dietz seit vielen Jahren zu den Kopf-Hals-Karzinomen und speziell zu HPV-assoziierten p16-positiven Oropharynxkarzinomen sowie möglichen Verbesserungen der Therapie und zum Erhalt der Lebensqualität der Tumorpatienten geforscht. In einer kürzlich erschienenen Kohortenstudie untersuchten die Wissenschaftler primär chirurgisch behandelte Patienten mit fortgeschrittenen p16-positiven Oropharynxkarzinomen mit Lymphknotenmetastasen. „Dabei haben wir die prognostischen Faktoren umfassend analysiert. Unsere Ergebnisse zeigen, dass einige prognostische Faktoren einen schlechteren Verlauf hinsichtlich der Wiederkehr der Krebserkrankung bedingen. Eine schlechtere Prognose haben Patienten mit p16-positiven Oropharynxkarzinomen, deren Lymphknotenmetastasen eine durch das Tumorwachstum zerstörte Kapsel aufwiesen, und diejenigen, bei denen der Subtyp HPV16 nicht nachgewiesen wurde", sagt Dr. Gunnar Wichmann, Laborleiter des HNO-Forschungslabors. Diese Arbeit zeige die Notwendigkeit auf, für eine korrekte Prognose sowohl die pathologische Untersuchung der Lymphknotenmetastasen auf Kapseldurchbruch wie auch den Nachweis von HPV16 einzubeziehen. Der Nachweis von p16-Positivität allein dürfe keinen Anlass zu einer veränderten Therapie geben.

Die Publikation wurde unter zentraler Beteiligung zweier junger Nachwuchswissenschaftlerinnen im Rahmen ihrer Promotionsprojek- te am Universitätsklinikum Leipzig erstellt. Beide Erstautorinnen haben inzwischen erfolgreich ihr Medizinstudium abgeschlossen und ihre Tätigkeit als Assistenzärztinnen am Evangelischen Krankenhaus Lutherstadt Wittenberg bzw. an der HNO-Klinik in Leipzig aufgenommen und schreiben aktuell an ihren Dissertationen.

Originalveröffentlichung in Cancer: Freitag J , Wald T, Kuhnt T, Gradistanac T, Kolb M, Dietz A, Wiegand S, Wichmann G. Extracapsular extension of neck nodes and absence of human papillomavirus 16-DNA are predictors of impaired survival in p16-positive oropharyngeal squamous cell carcinoma. Cancer 2020 May 1; 126(9): 1856-1872. DOI: 10.1002/cncr.32667

\section{Weitere Informationen:}

Prof. Dr. Andreas Dietz

Klinik und Poliklinik für Hals-, Nasen-, Ohrenheilkunde

Telefon: +49 341 97-21700

E-Mail: andreas.dietz@medizin.uni-leipzig.de Web: http://hno.uniklinikum-leipzig.de

Dr. Gunnar Wichmann

Klinik und Poliklinik für Hals-, Nasen-, Ohrenheilkunde

Laborleiter HNO-Forschungslabor

Telefon: +493419721926

E-Mail: Gunnar.Wichmann@medizin.uni-

leipzig.de

Quelle: Pressemitteilung der Universität Leipzig, www.uni-leipzig.de 